OPEN ACCESS

Edited by:

John Lui Yovich,

Pivet Medical Center, Australia

Reviewed by:

Kevin Noel Keane,

Curtin University, Australia

Xing Yang,

Sixth Affiliated Hospital of Sun Yat-Sen

University, China

*Correspondence:

Chii-Ruey Tzeng

tzengcr@tmu.edu.tw

Specialty section: This article was submitted to

Reproduction,

a section of the journa

Frontiers in Endocrinology

Received: 22 June 2019 Accepted: 05 December 2019 Published: 20 December 2019

Citation:

Lee Y-X, Shen M-S and Tzeng C-R (2019) Low Dose Growth Hormone Adjuvant Treatment With Ultra-Long Ovarian Stimulation Protocol in Poor

Responders Showed Non-inferior Pregnancy Outcome Compared With Normal Responders.

Front. Endocrinol. 10:892. doi: 10.3389/fendo.2019.00892

\section{Low Dose Growth Hormone Adjuvant Treatment With Ultra-Long Ovarian Stimulation Protocol in Poor Responders Showed Non-inferior Pregnancy Outcome Compared With Normal Responders}

\author{
Yi-Xuan Lee ${ }^{1,2}$, Meng-Shun Shen ${ }^{3}$ and Chii-Ruey Tzeng ${ }^{2,4 *}$ \\ ${ }^{1}$ Graduate Institute of Clinical Medicine, Taipei Medical University, Taipei, Taiwan, ${ }^{2}$ Division of Infertility, Department of \\ Obstetrics and Gynecology, Taipei Medical University Hospital, Taipei, Taiwan, ${ }^{3}$ IHMED Reproductive Medical Center, Taipei, \\ Taiwan, ${ }^{4}$ Department of Obstetrics and Gynecology, School of Medicine, College of Medicine, Taipei Medical University, \\ Taipei, Taiwan
}

Background: Growth hormone $(\mathrm{GH})$ has long been used as adjuvant treatment in ovarian stimulation for in vitro fertilization (IVF), especially in poor responder (PR) patients. However, its clinical efficacy remains unclear, and most studies are underpowered owing to their small sample size with different regimens.

Methods: Our study was divided into two parts. The first part was a parallel randomized, observational study in which 184 patients who fulfilled the criteria of poor ovarian response (POR) were enrolled and received ultra-long ovarian stimulation protocol with or without GH adjuvant therapy. For the second part, clinical data were retrospectively extracted from 163 patients classified as PRs who received $10 \mathrm{IU}$ GH adjuvant therapy and 157 patients classified as normal responders (NRs) who received the same IVF protocol treatment without GH adjuvant therapy.

Results: For the first part of the study, the ovarian response, the number of oocytes retrieved, and the number of available embryos transferred were all significantly higher in the $\mathrm{GH}(+)$ group than in the $\mathrm{GH}(-)$ group. The clinical pregnancy rate was significantly higher in the $\mathrm{GH}(+)$ group (31.9 vs. $16.7 \%, p=0.0168)$. The miscarriage rate did not differ significantly between the groups. The ongoing pregnancy rate was also significantly higher in the $\mathrm{GH}(+)$ group than in the $\mathrm{GH}(-)$ group (26.6 vs. $14.4 \%, p=0.0418)$. Logistic regression revealed that the chance of clinical pregnancy in the $\mathrm{GH}(+)$ group significant increased 2.34-fold in comparison with the $\mathrm{GH}(-)$ group $(p=0.018)$. Subgroup analysis showed that the chance of clinical pregnancy in the $\mathrm{GH}(+)$ group significantly increased 2.38 -fold $(p=0.034)$. The second part of the study showed no statistical difference between the PR with $\mathrm{GH}$ and the NR without $\mathrm{GH}$ groups regarding the implantation rate (15.6 vs. $19.8 \%, p=0.3254)$ and the clinical pregnancy rate (31.9 vs. $39.5 \%$, $p=0.1565)$. The NR without GH group showed insignificantly higher chance of clinical pregnancy $(\mathrm{OR}=1.39, p=0.157)$ compared with the PR with $\mathrm{GH}$ group. 


\begin{abstract}
Conclusion: Our results suggested that low-dose GH supplementation may improve ovarian response and pregnancy outcome in POR patients, particularly in patients younger than 40 years old. Moreover, the low-dose GH effect in POR patients resulted in non-inferior clinical pregnancy outcome compared with NRs.
\end{abstract}

Keywords: growth hormone, low dose, poor ovarian response, in vitro fertilization, adjuvant treatment, pregnancy outcomes

\section{INTRODUCTION}

Controlled ovarian hyperstimulation $(\mathrm{COH})$ has long been a crucial part of in vitro fertilization (IVF), along with the development of assisted reproductive technologies (ART). The goal of $\mathrm{COH}$ is to recruit multiple follicles and to obtain many mature oocytes to increase chances for conception (1). Poor ovarian response (POR) to ovarian stimulation indicates a reduction in follicular response and a reduced number of retrieved oocytes. Despite using different stimulation protocols and multiple treatment courses of IVF, the pregnancy outcome remains poor in POR patients, which is frustrating for both the patients and the clinicians.

The incidencevs of POR to ovarian stimulation reportedly ranges from 9 to $24 \%$ of IVF-embryo transfer (ET) cycles, according to various studies $(2,3)$. POR, or poor responders (PRs), remains a significant challenge for IVF practice owing to not only the heterogeneity of the pathophysiology but also the lack of general consensus in the definition of POR. The latter directly leads to poor literature quality with insufficient evidence to identify any particular intervention to improve outcomes in POR patients. The definition of POR was under debate without uniform agreement for decades until the European Society of Human Reproduction and Embryology conducted a consensus study and reached a definition for POR in 2011. This so-called Bologna criterion defines poor response in IVF as comprising at least two of the following three features: (i) advanced maternal age ( $\geq 40$ years) or any other risk factor for POR, (ii) previous POR ( $\leq 3$ oocytes with a conventional stimulation protocol), and (iii) an abnormal ovarian reserve test [antral follicular count $($ AFC $)<5-7$ follicles or anti-mullerian hormone $(\mathrm{AMH})<0.5-$ $1.1 \mathrm{ng} / \mathrm{ml}$. Two episodes of POR after maximal stimulation are sufficient to define a patient as a PR in the absence of advanced maternal age (4).

Numerous studies have been conducted using different interventions for the management of POR. Among them, the use of growth hormone $(\mathrm{GH})$ as an adjuvant treatment with gonadotropins to facilitate follicular development and ovulation induction was first introduced by Homburg et al. in 1988 (5). GH is an anabolic peptide hormone which functions to increase cell growth and proliferation, and it has been reported to modulate the action of follicle-stimulating hormone (FSH) by binding to $\mathrm{GH}$ receptors on granulosa cells to increase the synthesis of insulin-like growth factor-I (IGF-I). IGF-I augments the effect of gonadotropin action on both the granulosa and theca cells and plays an essential role in follicular development, oocyte maturation, and steroidogenesis. It can also improve follicular survival and granulosa cell proliferation by directly inhibiting follicle apoptosis (5-9). Nevertheless, results from previous studies are controversial regarding the effect of $\mathrm{GH}$ as an adjuvant therapy during $\mathrm{COH}$. $\mathrm{GH}$ has been demonstrated to increase the retrieved oocytes and improve embryo quality and pregnancy outcomes in several studies (10-19) and metaanalyses $(2,3,20-22)$. However, several clinical trials failed to demonstrate significant benefits regarding clinical pregnancy and live birth rates (23-27). The small sample size of the trials, inconsistency in the definition of POR, and different stimulation protocols with $\mathrm{GH}$ regimens all contributed to the bias of the outcomes. Therefore, the true value of GH adjuvant treatment remains elusive to date.

Very few reports are available that discuss the influence of GH dosage and regimen. Furthermore, no previous study using an ultra-long down-regulation protocol combined with $\mathrm{GH}$ adjuvant treatment has been reported. In our study, we aimed to investigate the efficacy of low-dose GH adjuvant treatment in $\mathrm{PR}$ patients compared with normal responders (NRs) using an ultra-long ovarian stimulation protocol.

\section{MATERIALS AND METHODS Study Period and Participants}

This parallel randomized, observational cohort study was conducted in a single IVF center in Taipei Medical University Hospital from January 2010 to October 2012.

The study was divided into two parts. First, enrolled patients were classified as PRs who had fulfiled at least two of the following criteria: (i) advanced maternal age ( $\geq 40$ years old) or any other risk factors for POR, (ii) previous episode of POR ( $\leq 3$ mature oocytes retrieved with a conventional stimulation protocol), and (iii) an abnormal ovarian reserve test (AFC < 5-7 follicles or AMH $<0.5-1.1 \mathrm{ng} / \mathrm{ml}$ ) between January 2010 and November 2011. Second, the data of patients who were classified as PRs who received GH adjuvant therapy and as NRs who received the same IVF treatment protocol without GH adjuvant therapy were collected between January 2012 and October 2012.

\section{Clinical Management}

To prevent possible bias from different physicians, all patients were handled by a single clinician. In addition, to eliminate bias from fresh vs. frozen embryo transfer, only the first IVF cycle with fresh embryo transfer (ET) within the study period was 
analyzed. All patients who participated in the study followed a gonadotropin-releasing hormone $(\mathrm{GnRH})$ agonist ultra-long IVF protocol. In brief, the patients received a half-dose one-shot long-acting $\mathrm{GnRH}$ agonist (leuprolide acetate, $1.88 \mathrm{mg}$ ) at cycle day $1-3$, followed by ovulation induction with gonadotropin starting between day 35 and 40. The dosage of gonadotropin was adjusted according to the ovarian response, which was monitored by transvaginal ultrasonography and serum hormone level. When two or more follicles reached a diameter of 17$18 \mathrm{~mm}, 6,500-10,000$ IU of human chorionic gonadotropin (hCG) was administered, and transvaginal oocyte retrieval was performed $34-36 \mathrm{~h}$ later. Fertilization was conducted by intracytoplasmic sperm injection (ICSI) $\sim 2 \mathrm{~h}$ later, after oocyte denudation. Fresh day 3 ET was performed with the best-quality blastomeres.

The first part of the study was a parallel, randomized study. Patients who fulfilled the abovementioned inclusion criteria were randomly allocated into two groups (named the $\mathrm{GH}(+)$ group and the $\mathrm{GH}(-)$ group) with simple randomization using a tossing coin method. Patients in the $\mathrm{GH}(+)$ group $(n=94)$ received co-treatment with GH adjuvant therapy (Saizen; Merck Serono) at a dosage of 4,4 , and $2 \mathrm{IU}$ for three successive days, along with the ovulation induction. The total GH dosage was 10 IU for each patient in the $\mathrm{GH}(+)$ group. Patients in the $\mathrm{GH}(-)$ group $(n=90)$ received the same IVF protocol without GH adjuvant therapy.

For the second part of the study, patients who were classified as PRs and received co-treatment with GH adjuvant therapy $(n=163)$ and NR patients $(n=157)$ who received the same IVF protocol without GH adjuvant therapy were enrolled.

\section{Data Analysis and Statistics}

Clinical parameters including age, AMH level, E2 level on the day of hCG administration, total gonadotropin dosage, mean number of oocytes retrieved, number of embryos transferred, embryo quality, and number of surplus embryos were recorded and analyzed. The main outcomes of the study were implantation rate, clinical pregnancy rate, miscarriage rate, and ongoing pregnancy rate. The implantation rate was calculated as the ratio of the number of gestational sacs to the number of embryos transferred. Clinical pregnancy was defined as the presence of a positive heartbeat in a healthy gestational sac, detected by transvaginal ultrasound 45 weeks after embryo transfer. Early miscarriage was defined as pregnancy loss before 12 weeks of gestation. Ongoing pregnancy was defined as viable pregnancy after 20 weeks of gestation.

Data are presented as mean and SD for quantitative variables and percentage for qualitative variables. The odds ratio (OR) and 95\% confidence interval were calculated for clinical pregnancy rate and miscarriage rate. Comparisons between groups were performed using the Student's $t$-test for quantitative variables and the chi-square test for qualitative variables. SPSS (IBM Statistics, ver. 25) was used for all statistical analyses. A $p<0.05$ was considered statistically significant.
TABLE 1 | Clinical parameters and outcomes of poor-responders with $\mathrm{GH}(+)$ and $\mathrm{GH}(-)$ groups.

\begin{tabular}{|c|c|c|c|}
\hline & GH (+) & GH (-) & $P$-value \\
\hline$N$ & 94 & 90 & \\
\hline $\mathrm{AMH}, \mathrm{ng} / \mathrm{ml}$ & $1.1 \pm 0.4$ & $1.1 \pm 0.9$ & 1.0000 \\
\hline Age, year & $38 \pm 3.4$ & $37.1 \pm 3.8$ & 0.0919 \\
\hline E2 level on hCG day, pg/ml & $679 \pm 459$ & $457 \pm 357$ & $0.0003^{\star}$ \\
\hline No. of oocytes retrieved & $5.5 \pm 3.3$ & $2.1 \pm 0.7$ & $<0.0001^{*}$ \\
\hline No. of embryos transfer & $2.6 \pm 0.9$ & $1.7 \pm 0.7$ & $<0.0001^{*}$ \\
\hline Clinical pregnancy, $n$ (\%) & $30 / 94$ (31.9\%) & $15 / 90(16.7 \%)$ & $0.0168^{\star}$ \\
\hline Miscarriage, $n(\%)$ & 5/30 (16.6\%) & 2/15 (13.3\%) & 0.7755 \\
\hline Ongoing pregnancy, $n$ (\%) & 25/94 (26.6\%) & $13 / 90(14.4 \%)$ & $0.0418^{\star}$ \\
\hline
\end{tabular}

RESULTS

\section{Outcomes of PR Patient With GH (+) and GH (-) Groups}

The clinical parameters and main outcomes in both groups are listed in Table 1. The baseline characteristics of both groups including age and AMH did not differ statistically. The E2 level on the hCG day was significantly higher in the GH $(+)$ group $(679 \pm 459$ vs. $457 \pm 357, p=0.0003)$. The number of oocytes retrieved was also significantly higher in the $\mathrm{GH}(+)$ group than in the GH (-) group (5.5 \pm 3.3 vs. $2.1 \pm 0.7, p<0.0001)$, both indicating a higher ovarian response to stimulation in the $\mathrm{GH}(+)$ group. The number of embryo transfer was significant higher in the $\mathrm{GH}(+)$ group than in the $\mathrm{GH}(-)$ group ( $2.6 \pm 0.9$ vs. 1.7 $\pm 0.7, p<0.0001)$. The clinical pregnancy rate was significantly higher in the $\mathrm{GH}(+)$ group than in the $\mathrm{GH}(-)$ group (31.9 vs. $16.7 \%, p=0.0168$; OR $=2.34, p=0.0177)$, and the number of ET cycles was significantly higher in the GH $(+)$ group $(2.6$ \pm 0.9 vs. $1.7 \pm 0.7, p<0.0001)$. The miscarriage rate did not differ significantly between the groups. The ongoing pregnancy rate was also significantly higher in the $\mathrm{GH}(+)$ group than in the $\mathrm{GH}(-)$ group (26.6 vs. $14.4 \%, p=0.0418$ ).

We further subdivided both patient cohorts by age below and above 40 years old. The results showed that the E2 level on the hCG day was significantly higher in the GH $(+)$ group $(619 \pm$ 317 vs. $449 \pm 366, p=0.0065$ ) in patients under 40 years old but not in patients older than 40 years $(685 \pm 408$ vs. $503 \pm 336, p$ $=0.0716)$. Logistic regression revealed that the chance of clinical pregnancy in the $\mathrm{GH}(+)$ group significantly increased 2.34-fold $(p=0.018)$ in univariable analysis and 2.52-fold $(p=0.011)$ in multivariable analysis, respectively (Table 2). Subgroup analysis showed that the chance of clinical pregnancy in the $\mathrm{GH}(+)$ group significantly increased 2.38 -fold $(p=0.034)$ in patients $<40$ years old, but not in patients more than 40 years old (OR $=3.23, p=0.17$; Table 3).

\section{Outcomes of PR Patients With GH and NR Patients Without GH}

We compared the clinical parameters and main outcomes of PRs with GH co-treatment and NRs without GH co-treatment 
TABLE 2 | Logistic regression with univariate and multivariate analysis of the pregnancy outcome for poor-responders.

\begin{tabular}{|c|c|c|c|c|c|c|}
\hline Variable & & $\begin{array}{c}\text { Clinical pregnancy } \\
\text { rate, } N(\%)\end{array}$ & \multicolumn{4}{|c|}{ Clinical pregnancy OR $(95 \% \mathrm{Cl})$} \\
\hline Growth Hormone & $\mathrm{GH}(+)$ & $30(31.9 \%)$ & $\begin{array}{c}2.34 \\
(1.16-4.74)\end{array}$ & $0.018^{\star}$ & $\begin{array}{c}2.52 \\
(1.23-5.16)\end{array}$ & $0.011^{*}$ \\
\hline & GH $(-)$ & 15 (16.7\%) & 1.00 & & 1.00 & \\
\hline Age & $<40$ & $36(28.8 \%)$ & $\begin{array}{c}2.25 \\
(1.00-5.04)\end{array}$ & 0.05 & $\begin{array}{c}2.46 \\
(1.08-5.62)\end{array}$ & $0.032^{*}$ \\
\hline
\end{tabular}

${ }^{*} p<0.05$.

TABLE 3 | Subgroup analysis of the pregnancy outcome for poor-responders.

\begin{tabular}{llccc}
\hline Variable & Subgroup & $\begin{array}{c}\text { Clinical pregnancy } \\
\text { rate, } \boldsymbol{N}(\%)\end{array}$ & $\begin{array}{c}\text { Clinical pregnancy } \\
\text { OR (95\% Cl) }\end{array}$ & P-value \\
\hline $\mathrm{GH}(+)$ & Age $<40$ & $23(37.7 \%)$ & $2.25(0.84-6.03)$ & 0.106 \\
& Age $\geq 40$ & $7(21.2 \%)$ & 1.00 & \\
$\mathrm{GH}(-)$ & Age $<40$ & $13(20.3 \%)$ & $3.06(0.63-14.64)$ & 0.162 \\
& Age $\geq 40$ & $2(7.7 \%)$ & 1.00 & \\
Age $<40$ & $\mathrm{GH}(+)$ & $23(37.7 \%)$ & $2.38(1.07-5.28)$ & $0.034^{\star}$ \\
& $\mathrm{GH}(-)$ & $13(20.3 \%)$ & 1.00 & \\
Age $\geq 40$ & $\mathrm{GH}(+)$ & $7(21.2 \%)$ & $3.23(0.61-17.10)$ & 0.17 \\
& $\mathrm{GH}(-)$ & $2(7.7 \%)$ & 1.00 & \\
\hline
\end{tabular}

${ }^{*} p<0.05$.

(Table 4). The PR with GH $[\mathrm{PR}+\mathrm{GH}(+)]$ patients were significantly older than the NR without GH $[\mathrm{NR}+\mathrm{GH}(-)]$ patients $(38.8 \pm 4.1$ vs. $35 \pm 3.8, p<0.0001)$, with significantly lower AMH $(1.6 \pm 1.4$ vs. $3.6 \pm 2.7, p<0.0001)$. During ovulation induction, the $\mathrm{PR}+\mathrm{GH}(+)$ patients required higher total gonadotropin ( $2495 \pm 915$ vs. $2160 \pm 513, p=0.0001)$, but had lower E2 level on hCG day ( $872 \pm 723.6$ vs. $1652 \pm$ $1141, p=0.0001)$ compared with the NR $+\mathrm{GH}(-)$ patients. The number of oocytes retrieved, the number of good-quality embryos, and the number of surplus frozen embryos were all significantly lower in the $\mathrm{PR}+\mathrm{GH}(+)$ group than in the NR $+\mathrm{GH}(-)$ group. However, there was no statistical difference between the two groups regarding the number of embryos transferred $(2.5 \pm 1.0$ vs. $2.6 \pm 1.8, p=0.5375)$, the implantation rate ( 15.6 vs. $19.8 \%, p=0.3254)$, and the clinical pregnancy rate (31.9 vs. $39.5 \%, p=0.1565$ ).

Logistic regression revealed that the chance of clinical pregnancy in patients $<40$ years old significantly increased 2.41 fold $(p=0.003)$ in univariable analysis and 2.33-fold ( $p=$ 0.006 ) in multivariable analysis, respectively (Table 5). The NR $+\mathrm{GH}(-)$ group showed a slightly higher chance of clinical pregnancy $(\mathrm{OR}=1.39, p=0.157$ in univariable; $\mathrm{OR}=1.08, p=$ 0.76 in multivariable analysis, respectively), but these differences were insignificant (Table 5). Subgroup analysis showed that the chance of clinical pregnancy in the $\mathrm{GH}(+)$ group significantly increased 2.13 -fold $(p=0.033)$ in patients $<40$ years old compared with patients more than 40 years old. The chance of clinical pregnancy showed an insignificant increase in patients $<40$ years old $(\mathrm{OR}=1.14, p=0.63)$ and an insignificant
TABLE 4 | Clinical parameters and outcomes of poor-responder with GH co-treatment and normal-responder without GH co-treatment groups.

\begin{tabular}{lccc}
\hline & PR with GH (+) & NR with GH (-) & P-value \\
\hline$N$ & 163 & 157 & \\
Age, years & $38.8 \pm 4.1$ & $35 \pm 3.8$ & $<0.0001^{*}$ \\
AMH, ng/ml & $1.6 \pm 1.4$ & $3.6 \pm 2.7$ & $<0.0001^{*}$ \\
Total FSH, IU & $2495 \pm 915$ & $2160 \pm 513$ & $0.0001^{*}$ \\
E2 level on hCG day, pg/ml & $872 \pm 723.6$ & $1652 \pm 1141$ & $0.0001^{*}$ \\
No. of oocytes retrieved & $5.8 \pm 4.1$ & $10.3 \pm 6.0$ & $<0.0001^{*}$ \\
No. of good embryos & $3.8 \pm 2.7$ & $7.0 \pm 4.3$ & $<0.0001^{*}$ \\
No. of embryos transfer & $2.5 \pm 1.0$ & $2.6 \pm 1.8$ & 0.5375 \\
No. of surplus embryos frozen & $0.7 \pm 1.7$ & $3.0 \pm 3.8$ & $<0.0001^{*}$ \\
Implantation rate & $15.6 \%$ & $19.8 \%$ & 0.3254 \\
Clinical pregnancy rate, $n(\%)$ & $52 / 163(31.9 \%)$ & $62 / 157(39.5 \%)$ & 0.1565 \\
\hline${ }^{*} p<0.05$. & & &
\end{tabular}

decrease in patients more than 40 years old $(\mathrm{OR}=0.78, p=$ 0.72) (Table 6).

\section{DISCUSSIONS}

The first part of our study demonstrated that, in POR patients, low-dose GH supplementation significantly increased ovarian response, the number of oocyte retrieved, clinical pregnancy rate, and the ongoing pregnancy rate. The number of embryo transferred was also significant increase, which was mainly due to more available embryos obtained after GH supplementation. These results echoed the conclusions of previous studies and several meta-analyses which reported increased ovarian response and pregnancy outcome after $\mathrm{GH}$ supplementation. Using logistic regression and subgroup analysis, our results demonstrated that the effect of $\mathrm{GH}$ seemed more prominent in younger patients $(<40$ years old).

In the second part of our study, the POR patients were assumed to have poor prognosis because they were apparently older and had a significantly lower ovarian reserve than the NRs. POR patients with low-dose GH supplementation required a higher gonadotropin dosage but ended up with lower ovarian response, smaller oocyte yields, fewer good-quality embryos, and fewer surplus frozen embryos. However, the implantation rates and clinical pregnancy rates were comparable between 
TABLE 5 | Logistic regression with univariate and multivariate analysis of pregnancy outcome for poor-responder (PR) with GH co-treatment and normal-responder (NR) without $\mathrm{GH}$ co-treatment groups.

\begin{tabular}{|c|c|c|c|c|c|c|}
\hline \multirow[t]{2}{*}{ Variable } & & \multirow{2}{*}{$\begin{array}{c}\text { Clinical pregnancy } \\
\text { rate, } N(\%)\end{array}$} & \multicolumn{4}{|c|}{ Clinical pregnancy OR $(95 \% \mathrm{Cl})$} \\
\hline & & & $\begin{array}{c}\text { Univariable } \\
\text { analysis (Crude OR) }\end{array}$ & $P$-value & $\begin{array}{l}\text { Multivariable analysis } \\
\text { (Adjusted OR) }\end{array}$ & $P$-value \\
\hline \multirow[t]{2}{*}{ Growth Hormone } & $\mathrm{PR}(N=163) \mathrm{GH}(+)$ & 52 (31.9\%) & 1.00 & & 1.00 & \\
\hline & $\mathrm{NR}(N=157) \mathrm{GH}(-)$ & $62(39.5 \%)$ & $\begin{array}{c}1.39 \\
(0.88-2.20)\end{array}$ & 0.157 & $\begin{array}{c}1.08 \\
(0.66-1.77)\end{array}$ & 0.76 \\
\hline \multirow[t]{2}{*}{ Age } & $<40(N=234)$ & 95 (40.6\%) & $\begin{array}{c}2.41 \\
(1.36-4.27)\end{array}$ & $0.003^{*}$ & $\begin{array}{c}2.33 \\
(1.27-4.29)\end{array}$ & $0.006^{\star}$ \\
\hline & $\geq 40(N=86)$ & $19(22.1 \%)$ & 1.00 & & 1.00 & \\
\hline
\end{tabular}

${ }^{*} p<0.05$.

TABLE 6 | Subgroup analysis of the pregnancy outcome for poor-responder (PR) with $\mathrm{GH}$ co-treatment and normal-responder (NR) without GH co-treatment groups.

\begin{tabular}{|c|c|c|c|c|}
\hline Variable & Subgroup & $\begin{array}{c}\text { Clinical pregnancy } \\
\text { rate, } N(\%)\end{array}$ & $\begin{array}{c}\text { Clinical pregnancy } \\
\text { OR }(95 \% \mathrm{Cl})\end{array}$ & $P$-value \\
\hline \multirow{2}{*}{$\begin{array}{l}\mathrm{PR}, \mathrm{GH}(+), \\
N=163\end{array}$} & Age $<40$ & $36(38.7 \%)$ & $2.13(1.06-4.28)$ & $0.033^{\star}$ \\
\hline & Age $\geq 40$ & $16(22.9 \%)$ & 1.00 & \\
\hline \multirow{2}{*}{$\begin{array}{l}\mathrm{NR}, \mathrm{GH}(-) \\
N=157\end{array}$} & Age $<40$ & $59(41.8 \%)$ & $3.12(0.85-11.43)$ & 0.086 \\
\hline & Age $\geq 40$ & $3(18.8 \%)$ & 1.00 & \\
\hline \multirow{2}{*}{$\begin{array}{l}\text { Age }<40 \\
N=234\end{array}$} & $\mathrm{PR}, \mathrm{GH}(+)$ & $36(38.7 \%)$ & 1.00 & 0.63 \\
\hline & $\mathrm{NR}, \mathrm{GH}(-)$ & $59(41.8 \%)$ & $1.14(0.67-1.95)$ & \\
\hline \multirow{2}{*}{$\begin{array}{l}\text { Age } \geq 40 \\
N=86\end{array}$} & $\mathrm{PR}, \mathrm{GH}(+)$ & $16(22.9 \%)$ & 1.00 & 0.72 \\
\hline & $\mathrm{NR}, \mathrm{GH}(-)$ & 3 (18.8\%) & $0.78(0.20-3.08)$ & \\
\hline
\end{tabular}

${ }^{*} p<0.05$.

POR patients with low-dose GH adjuvant treatment and NRs without GH.

$\mathrm{GH}$ exerts biological effects on most of the tissue, including tissue metabolism and induced local synthesis of IGF-I to facilitate cell growth (8). In ovarian function, $\mathrm{GH}$ is necessary for oogenesis and folliculogenesis, which is fundamental for optimal female fertility (28). In the second part of study, we believed that the effect of $\mathrm{GH}$ compensated the poorprognosis of this group and brought about comparable, or at least non-inferior, pregnancy outcomes compared with the NRs. Nevertheless, we could not rule out the beneficial effect of GH on other aspects, for example, the endometrium, that influenced pregnancy outcomes, since the NR group still possessed more oocytes and a higher embryo yield. The uterus is also a site of both GH production and GH action (28). The glandular cells of human endometrium and decidual tissue express $\mathrm{GH}$ receptors from the late luteal phase throughout pregnancy. Therefore, $\mathrm{GH}$ is speculated to play an important role in implantation (29). Previous studies also reported that GH might improve clinical outcome by increasing endometrial blood perfusion and improving endometrial receptivity during frozen-thawed ET in patients with repeated implantation failure $(30,31)$.
There is no standard protocol regarding $\mathrm{GH}$ regimen and dosage to date. The use of GH ranges from 4 to $24 \mathrm{IU}$, depending on different studies, and is usually started from previous cycle day 21 until the day of hCG administration. It is usually injected daily or on alternate days $(2,20,21)$. Most studies have shown a positive impact of $\mathrm{GH}$ on ovarian response and pregnancy outcome, without adverse effect reported. Nevertheless, GH may have a detrimental effect on insulin resistance, and high $\mathrm{GH}$ levels can inhibit fertility and promote neoplasm; the exact threshold dosage is still unclear (28). Moreover, an increased economic burden is inevitable since GH is very expensive. To our best knowledge, ours is only the second low-dose GH supplementation study in the literature. Lattes et al. (14) conducted a self-controlled study of 64 PRs who failed to reach pregnancy in the previous cycle and were the first to use a $\mathrm{GnRH}$ agonist long protocol co-treatment with low-dose $\mathrm{GH}$ supplementation (0.5 IU/day) from previous cycle day 21 until the day of hCG injection. The average day of $\mathrm{COH}$ was 11.2 days, and the estimated total dose of GH was about 9.5-10 IU, almost the same as in our study. However, in addition to a different study design, the regimen we used required injections for only 3 days. Furthermore, we used an ultra-long GnRH agonist protocol, which is less common worldwide. In fact, we believe that the ultra-long protocol is effective, flexible, and more patient-friendly when combined with long-acting gonadotropin to reduce the injection times.

We acknowledged that the limitations of our studies included a small sample size and the retrospective nature of the second part of the study. In addition, our results did not take into account the condition of the endometrium and other clinical parameters such as ploidy status, fertilization rate, and live birth rate. A randomized prospective clinical trial with a larger sample size is needed to validate the preliminary results.

In conclusion, our results suggested that low-dose $\mathrm{GH}$ supplementation may improve oocyte and embryo yields, clinical pregnancy rate, and ongoing pregnancy rate in POR patients, especially in patients younger than 40 years old. The effect of low-dose GH in POR patients resulted in non-inferior clinical pregnancy outcomes compared with NRs.

\section{DATA AVAILABILITY STATEMENT}

The datasets generated for this study are available on request to the corresponding author. 


\section{ETHICS STATEMENT}

The study involving human participants was reviewed and approved by the Joint Institutional Review Board of Taipei Medical University (TMU-JIRB, approval number: N201505056) for retrospective analysis and clinical data reporting. TMU-JIRB waived the requirement for further specific ethics approval or written informed consent since all procedures were not altered from routine clinical protocols, in accordance with the national legislation and the institutional requirements.

\section{AUTHOR CONTRIBUTIONS}

The present work was designed by C-RT. Data extraction and analysis were performed by Y-XL and M-SS. Patient recruitment

\section{REFERENCES}

1. Macklon NS, Stouffer RL, Giudice LC, Fauser BC. The science behind 25 years of ovarian stimulation for in vitro fertilization. Endocr Rev. (2006) 27:170-207. doi: 10.1210/er.2005-0015

2. Kolibianakis EM, Venetis CA, Diedrich K, Tarlatzis BC, Griesinger G. Addition of growth hormone to gonadotrophins in ovarian stimulation of poor responders treated by in-vitro fertilization: a systematic review and meta-analysis. Hum Reprod Update. (2009) 15:613-22. doi: 10.1093/humupd/dmp026

3. Kyrou D, Kolibianakis EM, Venetis CA, Papanikolaou EG, Bontis J, Tarlatzis BC. How to improve the probability of pregnancy in poor responders undergoing in vitro fertilization: a systematic review and meta-analysis. Fertil Steril. (2009) 91:749-66. doi: 10.1016/j.fertnstert.2007.12.077

4. Ferraretti AP, La Marca A, Fauser BC, Tarlatzis B, Nargund G, Gianaroli L, et al. ESHRE consensus on the definition of 'poor response' to ovarian stimulation for in vitro fertilization: the Bologna criteria. Hum Reprod. (2011) 26:1616-24. doi: 10.1093/humrep/der092

5. Homburg R, Eshel A, Abdalla HI, Jacobs HS. Growth hormone facilitates oculation induction by gonadotropins. Clin Endocrinol. (1988) 29:113-7. doi: 10.1111/j.1365-2265.1988.tb00252.x

6. Hsu C-J, Hammond JM. Concomitant effects of growth hormone on secretion of insulin-like growth factor i and progesterone by cultured porcine granulosa cells. Endocrinology. (1987) 121:1343-8. doi: 10.1210/endo-121-4-1343

7. Yoshimura Y, Nakamura Y, Koyama N, Iwashita M, Adachi T, Takeda Y. Effects of growth hormone on follicle growth, oocyte maturation, and ovarian steroidogenesis. Fertil Steril. (1993) 59:917-23. doi: 10.1016/S0015-0282(16)55882-8

8. Bachelot A, Monget $\mathrm{P}$, Imbert-Bollore $\mathrm{P}$, Coshigano $\mathrm{K}$, Kopchick JJ, Kelly PA, et al. Growth hormone is required for ovarian follicular growth. Endocrinology. (2002) 143:4104-12. doi: 10.1210/en.2002-2 20087

9. Regan SLP, Knight PG, Yovich JL, Arfuso F, Dharmarajan A. Growth hormone during in vitro fertilization in older women modulates the density of receptors in granulosa cells, with improved pregnancy outcomes. Fertil Steril. (2018) 110:1298-310. doi: 10.1016/j.fertnstert.2018.08.018

10. Owen EJ, West C, Mason BA, Jacobs HS. Co-treatment with growth hormone of sub-optimal responders in IVF-ET. Hum Reprod. (1991) 6:524-8. doi: 10.1093/oxfordjournals.humrep.a137372

11. Hazout A, Junca AM, Ménézo Y, de Mouzon J, Cohen-Bacrie P. Effect of growth hormone on oocyte competence in patients with multiple IVF failures. Reprod Biomed Online. (2009) 18:664-70. doi: 10.1016/S1472-6483(10)60011-9

12. Yovich JL, Stanger JD. Growth hormone supplementation improves implantation and pregnancy productivity rates for poor-prognosis was undertaken by C-RT. The initial manuscript draft was prepared by Y-XL and subsequently revised by Y-XL and C-RT. All authors approved the final submitted version.

\section{FUNDING}

This study was partially supported by research grants MOST-1062314-B-038-072 from the Ministry of Science and Technology, Taiwan, Academia Sinica. The funders played no role in the conduct of the study or the writing of the manuscript.

\section{ACKNOWLEDGMENTS}

The authors thank all the embryologists and nurses of the Center for Reproductive Medicine of Taipei Medical University Hospital for embryo culture, data recording, and other clinical assistance.

patients undertaking IVF. Reprod Biomed Online. (2010) 21:37-49. doi: 10.1016/j.rbmo.2010.03.013

13. Hu Z-P, Wang Y, Du X-G, Li R, Chen X-N, Wang H-Y, et al. Effects of growth hormone supplementation in patients undergoing IVF/ICSI-ET with poor ovarian response to gonadotropin. J Reprod Contracept. (2014) 25:32-40. doi: 10.7669/j.issn.1001-7844.2014.01.0032

14. Lattes K, Brassesco M, Gomez M, Checa MA. Low-dose growth hormone supplementation increases clinical pregnancy rate in poor responders undergoing in vitro fertilisation. Gynecol Endocrinol. (2015) 31:565-8. doi: 10.3109/09513590.2015.1025378

15. Ou J, Xing W, Li T, Zhu J, Zhu W. A preliminary observation on efficacy of growth hormone on poor ovarian responders in IVF/ICSI cycles. J Reprod Contracept. (2016) 27:12-7. doi: 10.7669/j.issn.1001-7844.2016.01.0012

16. Ho YK, Lee TH, Lee CI, Cheng EH, Huang CC, Huang LS, et al. Effects of growth hormone plus gonadotropins on controlled ovarian stimulation in infertile women of advanced age, poor responders, and previous in vitro fertilization failure patients. Taiwan J Obstet Gynecol. (2017) 56:806-10. doi: 10.1016/j.tjog.2017.10.018

17. Keane KN, Yovich JL, Hamidi A, Hinchliffe PM, Dhaliwal SS. Singlecentre retrospective analysis of growth hormone supplementation in IVF patients classified as poor-prognosis. BMJ Open. (2017) 7:e018107. doi: 10.1136/bmjopen-2017-018107

18. Ob'edkova K, Kogan I, Krikheli I, Dzhemlikhanova L, Muller V, Mekina I, et al. Growth hormone co-treatment in IVF/ICSI cycles in poor responders. Gynecol Endocrinol. (2017) 33(suppl. 1):15-7. doi: 10.1080/09513590.2017.1399693

19. Chu K, Pang W, Sun N, Zhang Q, Li W. Outcomes of poor responders following growth hormone co-treatment with IVF/ICSI mild stimulation protocol: a retrospective cohort study. Arch Gynecol Obstet. (2018) 297:131721. doi: 10.1007/s00404-018-4725-5

20. Duffy JM, Ahmad G, Mohiyiddeen L, Nardo LG, Watson A. Growth hormone for in vitro fertilization. Cochrane Database Syst Rev. 2010:CD000099. doi: 10.1002/14651858.CD000099.pub3

21. Li XL, Wang L, Lv F, Huang XM, Wang LP, Pan Y, et al. The influence of different growth hormone addition protocols to poor ovarian responders on clinical outcomes in controlled ovary stimulation cycles: a systematic review and meta-analysis. Medicine. (2017) 96:e6443. doi: 10.1097/MD.0000000000006443

22. de Ziegler D, Streuli I, Meldrum DR, Chapron C. The value of growth hormone supplements in ART for poor ovarian responders. Fertil Steril. (2011) 96:1069-76. doi: 10.1016/j.fertnstert.2011.09.049

23. Kucuk T, Kozinoglu H, Kaba A. Growth hormone co-treatment within a GnRH agonist long protocol in patients with poor ovarian response: a prospective, randomized, clinical trial. J Assist Reprod Genet. (2008) 25:123-7. doi: 10.1007/s10815-008-9212-7 
24. Eftekhar M, Aflatoonian A, Mohammadian F, Eftekhar T. Adjuvant growth hormone therapy in antagonist protocol in poor responders undergoing assisted reproductive technology. Arch Gynecol Obstet. (2013) 287:1017-21. doi: 10.1007/s00404-012-2655-1

25. Bassiouny YA, Dakhly DMR, Bayoumi YA, Hashish NM. Does the addition of growth hormone to the in vitro fertilization/intracytoplasmic sperm injection antagonist protocol improve outcomes in poor responders? A randomized, controlled trial. Fertil Steril. (2016) 105:697-702. doi: 10.1016/j.fertnstert.2015.11.026

26. Dakhly DMR, Bassiouny YA, Bayoumi YA, Hassan MA, Gouda HM, Hassan AA. The addition of growth hormone adjuvant therapy to the long down regulation protocol in poor responders undergoing in vitro fertilization: randomized control trial. Eur J Obst Gynecol Reprod Biol. (2018) 228:161-5. doi: 10.1016/j.ejogrb.2018.06.035

27. Norman RJ, Alvino H, Hull LM, Mol BW, Hart RJ, Kelly T-L, et al. Human growth hormone for poor responders: a randomized placebo-controlled trial provides no evidence for improved live birth rate. Reprod Biomed Online. (2019) 38:908-15. doi: 10.1016/j.rbmo.2019.02.003

28. Hull KL, Harvey S. Growth hormone and reproduction: a review of endocrine and autocrine/paracrine interactions. Int J Endocrinol. (2014) 2014:234014. doi: 10.1155/2014/234014

29. Sbracia M, Scarpellini F, Poverini R, Alò PL, Rossi G, Di Tondo U. Immunohistochemical localization of the growth hormone in human endometrium and decidua. Am J Reprod Immunol. (2004) 51:112-6. doi: 10.1046/j.8755-8920.2003.00127.x

30. Xue-Mei W, Hong J, Wen-Xiang Z, Yang L. The effects of growth hormone on clinical outcomes after frozen-thawed embryo transfer. Int J Gynaecol Obstet. (2016) 133:347-50. doi: 10.1016/j.ijgo.2015. 10.020

31. Altmae S, Mendoza-Tesarik R, Mendoza C, Mendoza N, Cucinelli F, Tesarik J. Effect of growth hormone on uterine receptivity in women with repeated implantation failure in an oocyte donation program: a randomized controlled trial. J Endocr Soc. (2018) 2:96-105. doi: 10.1210/js.201 7-00359

Conflict of Interest: The authors declare that the research was conducted in the absence of any commercial or financial relationships that could be construed as a potential conflict of interest.

Copyright (c) 2019 Lee, Shen and Tzeng. This is an open-access article distributed under the terms of the Creative Commons Attribution License (CC BY). The use, distribution or reproduction in other forums is permitted, provided the original author(s) and the copyright owner(s) are credited and that the original publication in this journal is cited, in accordance with accepted academic practice. No use, distribution or reproduction is permitted which does not comply with these terms. 